\title{
(Part of) the Case for a Pragmatic Approach to Validity: Comment on De Houwer, Teige-Mocigemba, Spruyt, and Moors (2009)
}

\author{
Brian A. Nosek \\ University of Virginia
}

\author{
Anthony G. Greenwald \\ University of Washington
}

\begin{abstract}
In their review of validity of the Implicit Association Test and affective priming, J. De Houwer, S. Teige-Mocigemba, A. Spruyt, and A. Moors (2009) identified validity with establishment of "basic theoretical understanding" of the measures. It is agreed that theoretical understanding has an important role in making measures more valid and useful. Nevertheless, the authors conclude that scientific advancement will more often be well served by prioritizing pragmatic goals of establishing the predictive validity of the measures and their adequate sensitivity to individual differences.
\end{abstract}

Keywords: validity, individual differences, Implicit Association Test, implicit measures, implicit social cognition

De Houwer, Teige-Mocigemba, Spruyt, and Moors (2009) reviewed the research literature concerning two procedures: the Implicit Association Test (IAT; Greenwald, McGhee, \& Schwartz, 1998) and affective priming (AP; Fazio, Sanbonmatsu, Powell, \& Kardes, 1986). Their aims were to describe the attributes that cause variation in IAT and AP effects and to evaluate the extent to which these measures are justifiably characterized as implicit.

The major portion of De Houwer et al.'s (2009) review evaluated the evidence bearing on theoretical interpretation of IAT and AP procedures. In doing this, the authors answered a "what" or construct identity question (what psychological attributes contribute to the measured effects) and a "how" or process question (what processes connect the psychological attributes to the measured effects). Their review effectively assembled theretofore scattered contributions to theoretical understanding of IAT and AP measurement procedures. There have been brief reviews of portions of this material in some overviews of the IAT (Greenwald \& Nosek, 2001; Lane, Banaji, Nosek, \& Greenwald, 2007; Nosek, Greenwald, \& Banaji, 2007) but nothing as comprehensive as the review by De Houwer et al.

There are a few issues on which we found ourselves disagreeing with De Houwer et al.'s (2009) interpretation of the available evidence. Those disagreements mostly involved questions that presently lack definitive answers and that De Houwer et al. appropriately characterized as unresolved. We feel no need to advance or develop our preferred theoretical interpretations of those points in this article. Instead, we focus on consideration of De Houwer et al.'s criteria for drawing conclusions about the validity of implicit measures.

Brian A. Nosek, Department of Psychology, University of Virginia; Anthony G. Greenwald, Department of Psychology, University of Washington.

This research was supported by the National Science Foundation (REC0634041) and by the Implicit and Unconscious Cognition Research Fund at the University of Washington.

Correspondence concerning this article should be sent to Brian A. Nosek, Department of Psychology, 102 Gilmer Hall, University of Virginia, Charlottesville, VA 22904-4400. E-mail: nosek@virginia.edu
We make three points: (a) De Houwer et al.'s (2009) criterion for validity (derived from Borsboom, Mellenbergh, \& van Heerden, 2004) — that validity consists of understanding "how the attribute causes the measurement outcome"-is narrower than desirable; (b) there are reasonable alternatives to De Houwer et al.'s proposal to equate "implicit" with "automatic"; and (c) a pragmatic approach to validity (establishing that implicit measures can be used effectively) is more achievable than is De Houwer et al.'s approach of describing how implicit measures are interpreted conceptually.

\section{Misplaced Focus?}

De Houwer et al. (2009) drew on the conception of validity expressed in Borsboom et al. (2004): "a test is valid for measuring an attribute if and only if (a) the attribute exists and (b) variations in the attribute causally produce variations in the outcomes of the measurement procedure" (p. 1061). As explained by De Houwer et al., Borsboom et al.'s "realist" approach led De Houwer et al. to focus on discerning (a) the psychological attributes influencing measures that thereby deserve to be called implicit and (b) the process or processes by which those attributes lead to the effects. In the case of the IAT, De Houwer et al. sought to choose between deciding whether the attribute (the "what") tapped by the IAT is more properly understood as association strength or similarity and whether the process (the "how") of the IAT is more properly characterized as diffusion (random walk), response activation, or task switching.

De Houwer et al.'s (2009) consideration of the relative merits of association strength versus similarity as the identity of the psychological attribute underlying the IAT can be used to explain why we believe that such attribute identification is not the most useful focus of validity research. In identifying association as the attribute underlying the IAT, Greenwald, Nosek, Banaji, and Klauer (2005) described a conception of association that was "not tied to any specific theory of the structure of associative mental representations" (p. 421). Drawing on classic interpretations of association by Aristotle, Hume, and others, Greenwald et al. observed that 
similarity was understood, in those classic views, as one of several sources of association strength (p. 421). Their "theoryuncommitted" conception of association thus bypassed debate over whether the attribute underlying the IAT should be conceived of as association strength versus similarity. In their interpretation, those two views were, conceptually, part of a single interpretation.

Another problem that we see with De Houwer et al.'s (2009) equation of validity with a specific psychological interpretation is that understanding of psychological attributes undergoes evolutionary changes as research evidence accumulates. That is why Greenwald et al. (2005), Rothermund and Wentura (2004), and De Houwer et al. were not obliged to (and indeed did not) use the same operational definitions of association, similarity, and salience in their treatments of those conceptual interpretations. Because of these allowable variations in operational definitions of concepts, debates such as the one framed by De Houwer et al.- concerning association versus similarity as the attribute measured by the IAT - have the undesirable property of potentially being sustained indefinitely without resolution. That is, by use of different operations to identify the same concept, different researchers can interpret a single body of evidence as both supporting and rejecting the same conceptual interpretation.

There are many examples in psychology's history of conceptual debates being waged for decades without the clear emergence of a winning side. One example is the still-continuing debate, which started in 1956 (Nowlis \& Nowlis, 1956), over whether affective valence should be conceived of as a single bipolar dimension or as separable dimensions of positive and negative affect. Antagonists in such long-unresolved debates very often agree in their description of the empirical phenomena and disagree only on how to name the underlying psychological attributes. We worry that such (apparently) unproductive debates over conceptual identification of attributes underlying psychological measures will be sustained by De Houwer et al.'s (2009) approach.

\section{What Is Implicit?}

The term implicit entered cognitive psychology through the research and writing of Graf and Schacter (1985) and Schacter (1987) on the topic of implicit memory. Those researchers adopted implicit as an appropriate descriptor for a growing collection of work on "remembering without awareness" (Jacoby \& Witherspoon, 1982). In describing implicit memory in the review article that ultimately established broad use of that term, Schacter (1987) described the contrast between implicit and explicit memory and observed that "these are descriptive [italics added] concepts that are primarily concerned with a person's psychological experience at the time of retrieval" (p. 501). In these introductory publications, the word implicit was used to describe an effect that occurred without memory for its cause.

In extending the term implicit into social psychology, Greenwald and Banaji (1995) deliberately borrowed from the cognitive psychologists' usage. As they stated, "The signature of implicit cognition is that traces of past experience affect some performance, even though the influential earlier experience is not remembered in the usual sense-that is, it is unavailable to selfreport or introspection" (p. 4).

This extremely brief history makes it clear that implicit entered cognitive and social psychology as a descriptive term, and it is still used by many in that fashion. De Houwer et al. (2009) suggested that "the term implicit can best be understood as being synonymous with the term automatic" (p. 350), and in this context they alluded to discussions that identify multiple aspects of automaticity (e.g., lack of intention, lack of awareness, lack of control, efficiency).

If De Houwer et al. (2009) indeed regard implicit and automatic as synonyms, then they might forgo one of the two terms, so that implicit could retain its broader descriptive understanding that maps well onto past usages. In our understanding of current research practices, a measure can be called implicit if it does not require awareness of the relation of the attribute to the response. Although awareness is not required for implicit measurement, this definition does not require that this awareness be absent.

From our perspective, an implicit measure can involve automatic processes, but we do not require an implicit measure to be composed entirely of automatic processes. We also think that virtually no measure used in human behavioral research is composed entirely of, or is entirely free of, automatic processes (cf. Jacoby, Lindsay, \& Toth, 1992; Reingold \& Merikle, 1988). If these assumptions are correct, it is difficult to treat automaticity as a sine qua non for establishing that a measure is implicit, as all measures will include automatic and nonautomatic process components. De Houwer et al.'s (2009) intent might be to conceive of the implicitness of a measure as proportional to the extent to which the measure's process components are automatic, but that would make it difficult for them to achieve their apparent goal of categorically distinguishing implicit from nonimplicit measures.

Implicit measures may diverge from self-reports of experiences because respondents are unable or unwilling to reveal those experiences via self-report procedures. In most discussions, including De Houwer et al.'s (2009), inability to report refers to a person's lack of awareness of some aspect of the attribute, the response, or the link between attribute and response.

Another aspect of inability implies neither unawareness nor automaticity. People may be unable to report about an attribute because they do not understand or do not know how to communicate its meaning, even though they are aware of possessing this meaning. Such inability is particularly evident for nonlinguistically represented information and for thoughts that might produce a palpable experience but are too complex to report. IAT procedures are already used with very young children to obtain useful measures under these circumstances. With appropriate ingenuity, these procedures might even be adapted for other species.

Lack of capacity to report on mental contents, or lack of understanding how to translate a mental content into a requested response format, prevents self-report measurement procedures from capturing the complete range of mental experience. This feature of inability is not part of the concept "automaticity," but, we suggest, it is nevertheless an important aspect of the concept "implicit." In sum, because (a) no measure is composed entirely of automatic processes and (b) there are features of implicit that (in our view) are not shared with automatic, we conclude that implicit is a distinct descriptive concept that is not a synonym of automatic.

\section{Realism versus Pragmatism}

Is it more important to define implicit in terms of attributes and processes or to interpret the value of implicit measures in terms of more practical considerations? De Houwer et al. (2009) favor the former approach. We favor the latter. 
De Houwer et al. (2009) correctly noted that "psychological attributes ... cannot be observed directly" (p. 349). This wellrecognized unobservability of psychological attributes has implications for understanding the claim (of De Houwer et al. and Borsboom et al., 2004) that "the [measured] attribute exists." De Houwer et al. explicitly refrained from making any ontological assumptions about what exists means in this case. In our view, the existence assumption is sustainable only if it is equated with the materialist assumption that all phenomena are the result of material interactions that, presumably, involve the neural operations of the brain. Modern psychology is firmly rooted in materialism (Dennett, 1991; Fodor, 1974; Kim, 1994; Quine, 1951; Searle, 1995). In other words, it is not a claim that the only psychological attributes that can be validated are those that are physical entities or are observable if one has the right methods of measurement. De Houwer et al. do not take a position, but we believe that clarifying this assumption is important for understanding their conception of validity.

Borsboom et al. (2004) argued that "if one does not have an idea of how the attribute variations produce variations in measurement outcomes, one cannot have a clue as to whether the test measures what it should measure" (p. 1068). De Houwer et al. (2009) endorsed Borsboom's view of validity, stating "it should be clear... how these attributes causally produce the measurement outcome" (p. 350). We agree that understanding how attributes cause effects is important. Indeed, De Houwer et al.'s review illustrated how much has been learned about the IAT and AP procedures through experimental investigations. We suggest, however, that validation-at least in a practical sense-does not require that the underlying processes be understood. For example, even though the neural operations that translate mental representations into performance on self-report measures remain intractable in current psychological theory and evidence, many self-report procedures are widely regarded as having been sufficiently validated for scientific use. Also, for the past 15 years, psychologists have made extensive use of functional magnetic resonance imaging, though they acknowledge that the resulting measure is not of a psychological process at all but is, rather, an indicator of blood flow in the brain. They further acknowledge that there is no established understanding of the relationship between measured blood flow and neural activity (e.g., Boynton \& Finney, 2003).

Further, in the context of De Houwer et al.'s (2009) assumption that the attribute exists, many measures that we can conclude are useful-primarily because they are widely used-are ones that make no pretense to map onto psychological attributes. The dimensions of personality inventories such as the Minnesota Multiphasic Personality Inventory or the Big Five are not attributes residing in the heads of the people who are characterized by scores on these dimensions. Rather, these dimensions are abstractions that encompass many individuals. For the same reason, it seems inappropriate to say that the traits characterized by scores on these dimensions are well-defined attributes of the people so characterized. For example, the level of introversion of a respondent with a high score on a personality measure of that trait is defined more by a comparison of that person with others than by the level's being an identifiable attribute of the person. ${ }^{1}$

Finally, other widely used measures may not even be based on an attempt to obtain data from the person being characterized. Consider, for example, the many measures that are made by observers who judge sound or image recordings of persons. This is also true of widely used student ratings that are designed to evaluate an instructor's teaching ability. (However, we regard the measured attribute as having been misidentified in this case, because correlational validity studies show that student ratings primarily assess instructor likability rather than teaching ability; see Greenwald, 1997.)

Two conclusions are implied by these examples of measures that are used effectively even in the absence of an understanding of the attributes being measured or the process by which the measure is generated. First, we conclude that Borsboom et al.'s (2004) conception of validity, which was adopted by De Houwer et al. (2009), does not descriptively characterize the preponderance of scientific work in psychology. Second, we are not persuaded of De Houwer et al.'s view that Borsboom et al.'s analysis should be treated as normative (i.e., "that it clarifies what researchers should [italics added] aim for when developing implicit measures"; p. 362). In our view, the most important considerations in appraising validity of psychological measures are those that speak to the measures' usefulness in research and application, in particular predictive validity and psychometric properties that appraise the ability of implicit measures to reliably characterize individual differences. If De Houwer et al. strongly sustain Borsboom et al.'s claim that validity depends on definitive identification of the causal relationship between attributes and outcomes, De Houwer et al. might be obliged to conclude that modern psychology currently has no valid measurement procedures. In that respect, it is noteworthy that De Houwer et al. did not provide any examples of measures that attain their validity criteria.

As a final observation in this comment, we note our strong agreement with De Houwer et al.'s (2009) concluding observation that

without a basic level of theoretical understanding of the measures, there is little ground for predicting when a measure will be related to which kind of behavior. Progress in obtaining evidence for relations between measures and behavior will thus proceed slowly and haphazardly. Likewise, there will be little guidance for attempts to improve [italics added] the quality of the measures. (p. 364)

This observation, which asserts that theoretical understanding of a measure is needed to improve the measure's quality, may seem puzzling. If De Houwer et al. (2009) understand quality (i.e., validity) of a measure as fully contained in theoretical understanding of the measure, what aspect of quality remains to be improved once the theoretical interpretation has been established? We read this statement as De Houwer et al.'s tacit acknowledgment that quality of a measure indeed requires more than theoretical understanding. And we strongly believe that achieving such other indicators of quality (e.g., predictive validity and measurement reliability) will be advanced by psychological theoretical understanding of the performances that produce the measure.

\footnotetext{
${ }^{1}$ Borsboom et al. (2004) claimed to have the means of dealing with "interindividual" measurement structures and observed that "the only thing that can cause variation over people is something that also varies over people" (p. 1069). However, this assertion is left undeveloped, both in the 2004 article and in Borsboom, Mellenbergh, and van Heerden (2003).

\section{References}

Borsboom, D., Mellenbergh, G. J., \& van Heerden, J. (2003). The theoretical status of latent variables. Psychological Review, 110, 203-219.
} 
Borsboom, D., Mellenbergh, G. J., \& van Heerden, J. (2004). The concept of validity. Psychological Review, 111, 1061-1071.

Boynton, G. M., \& Finney, E. M. (2003). Orientation-specific adaptation in human visual cortex. Journal of Neuroscence, 23, 8781-8787.

De Houwer, J., Teige-Mocigemba, S., Spruyt, A., \& Moors, A. (2009). Implicit measures: A normative analysis and review. Psychological Bulletin, 135, 347-368.

Dennett, D. (1991). Consciousness explained. Boston: Little, Brown.

Fazio, R. H., Sanbonmatsu, D. M., Powell, M. C., \& Kardes, F. R. (1986). On the automatic activation of attitudes. Journal of Personality and Social Psychology, 50, 229-238.

Fodor, J. A. (1974). Special sciences. Synthese, 28, 97-115.

Graf, P., \& Schacter, D. L. (1985). Implicit and explicit memory for new associations in normal and amnesic subjects. Journal of Experimental Psychology: Learning, Memory, and Cognition, 11, 501-518.

Greenwald, A. G. (1997). Validity concerns and usefulness of student ratings. American Psychologist, 52, 1182-1186.

Greenwald, A. G., \& Banaji, M. R. (1995). Implicit social cognition: Attitudes, self-esteem, and stereotypes. Psychological Review, 102, $4-27$.

Greenwald, A. G., McGhee, D. E., \& Schwartz, J. L. K. (1998). Measuring individual differences in implicit cognition: The Implicit Association Test. Journal of Personality and Social Psychology, 74, 1464-1480.

Greenwald, A. G., \& Nosek, B. A. (2001). Health of the Implicit Association Test at age 3. Zeitschrift für Experimentelle Psychologie, 48, 85-93.

Greenwald, A. G., Nosek, B. A., Banaji, M. R., \& Klauer, K. C. (2005). Validity of the salience asymmetry interpretation of the IAT: Comment on Rothermund and Wentura (2004). Journal of Experimental Psychology: General, 134, 420-425.

Jacoby, L. L., Lindsay, D. S., \& Toth, J. P. (1992). Unconscious influences revealed: Attention, awareness, and control. American Psychologist, 47, 802-809.
Jacoby, L. L., \& Witherspoon, D. (1982). Remembering without awareness. Canadian Journal of Psychology/Revue canadienne de psychologie, 36, 300-324.

Kim, J. (1994). Multiple realization and the metaphysics of reduction. Philosophy and Phenomenological Research, 52, 1-26.

Lane, K. A., Banaji, M. R., Nosek, B. A., \& Greenwald, A. G. (2007). Understanding and using the Implicit Association Test: IV: Procedures and validity. In B. Wittenbrink \& N. Schwarz (Eds.), Implicit measures of attitudes: Procedures and controversies (pp. 59-102). New York: Guilford Press.

Nosek, B. A., Greenwald, A. G., \& Banaji, M. R. (2007). The Implicit Association Test at age 7: A methodological and conceptual review. In J. A. Bargh (Ed.), Automatic processes in social thinking and behavior (pp. 265-292). Hove, England: Psychology Press.

Nowlis, V., \& Nowlis, H. H. (1956). The description and analysis of mood. Annals of the New York Academy of Sciences, 65, 345-355.

Quine, W. V. O. (1951). Two dogmas of empiricism. Philosophical Review, 60, 20-43.

Reingold, E. M., \& Merikle, P. M. (1988). Using direct and indirect measures to study perception without awareness. Perception and Psychophysics, 44, 563-575.

Rothermund, K., \& Wentura, D. (2004). Underlying processes in the Implicit Association Test (IAT): Dissociating salience from associations. Journal of Experimental Psychology: General, 133, 139-165.

Schacter, D. L. (1987). Implicit memory: History and current status. Journal of Experimental Psychology: Learning, Memory, and Cognition, 13, 501-518.

Searle, J. (1995). The construction of social reality. New York: Free Press.

Received November 16, 2008

Revision received November 26, 2008

Accepted December 1, 2008 